\title{
Wide-field surveys from the SNAP mission
}

\section{Alex G. Kim, Carl W. Akerlof, Greg Aldering, R. Amanullah, Pierre Astier, et al.}

Alex G. Kim, Carl W. Akerlof, Greg Aldering, R. Amanullah, Pierre Astier, E. Barrelet, Christopher Bebek, Lars Bergstrom, J. Bercovitz, Gary M. Bernstein, M. Bester, A. Bonissent, C. Bower, William C. Carithers, Eugene D. Commins, C. Day, Susana E. Deustua, R. DiGennaro, A. Ealet, Richard S. Ellis, M. Eriksson, Andrew Fruchter, JeanFrancois Genat, Gerson Goldhaber, Ariel Goobar, Donald E. Groom, Stewart E. Harris, Peter R. Harvey, Henry D. Heetderks, Steven E. Holland, Dragan Huterer, Armin Karcher, William F. Kolbe, B. Krieger, Robin E. Lafever, J. Lamoureux, Michael L. Lampton, Michael E. Levi, Daniel S. Levin, Eric V. Linder, Stewart C. Loken, Roger Malina, R. Massey, Timothy McKay, Shawn P. McKee, Ramon Miquel, E. Mortsell, N. Mostek, Stuart Mufson, J. A. Musser, Peter E. Nugent, Hakeem M. Oluseyi, Reynald Pain, Nicholas P. Palaio, David H. Pankow, Saul Perlmutter, R. Pratt, Eric Prieto, Alexandre Refregier, Jason Rhodes, Kem Edward Robinson, N. Roe, Michael Sholl, Michael S. Schubnell, G. Smadja, George F. Smoot, Anthony Spadafora, Gregory Tarle, Andrew D. Tomasch, H. von der Lippe, D. Vincent, J.-P. Walder, Guobin Wang, "Wide-field surveys from the SNAP mission," Proc. SPIE 4836, Survey and Other Telescope Technologies and Discoveries, (24 December 2002); doi: $10.1117 / 12.457953$ 


\title{
Wide-Field Surveys from the SNAP Mission
}

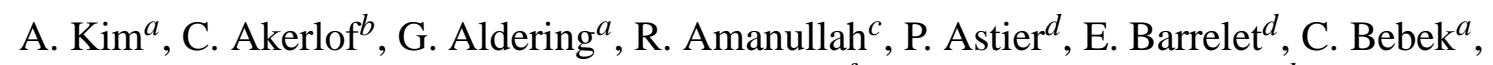
L. Bergström ${ }^{c}$, J. Bercovitz $^{a}$, G. Bernstein ${ }^{e}$, M. Bester ${ }^{f}$, A. Bonissent ${ }^{g}$, C. Bower ${ }^{h}$, W. Carithers ${ }^{a}$,

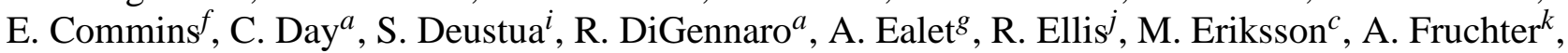

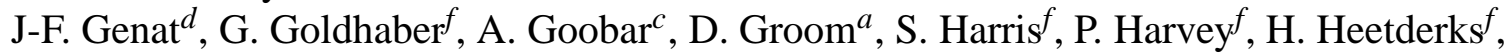
S. Holland ${ }^{a}$, D. Huterer ${ }^{l}$, A. Karcher ${ }^{a}$, W. Kolbe ${ }^{a}$, B. Krieger ${ }^{a}$, R. Lafever ${ }^{a}$, J. Lamoureux $^{a}$, $^{b}$

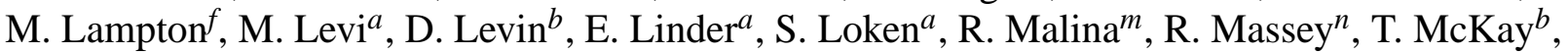
S. McKee ${ }^{b}$, R. Miquel ${ }^{a}$, E. Mörtsell ${ }^{c}$, N. Mostek ${ }^{h}$, S. Mufson ${ }^{h}$, J. Musser $^{h}$, P. Nugent $^{a}$, H. Oluseyi $^{a}$,

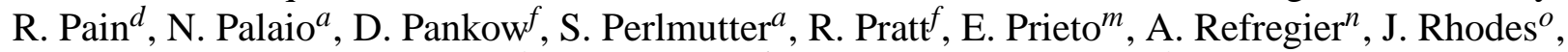

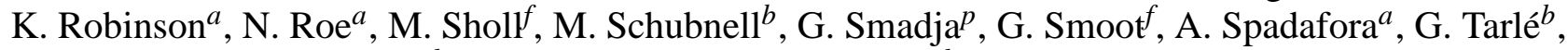

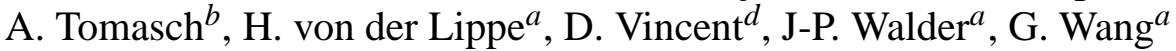

${ }^{a}$ Lawrence Berkeley National Laboratory, Berkeley CA, USA

${ }^{b}$ University of Michigan, Ann Arbor MI, USA

${ }^{c}$ University of Stockholm, Stockholm, Sweden ${ }^{d}$ CNRS/IN2P3/LPNHE, Paris, France

${ }^{e}$ University of Pennsylvania, Philadelphia PA, USA

$f_{\text {University of California, Berkeley CA, USA }}$

${ }^{g} \mathrm{CNRS} / \mathrm{IN} 2 \mathrm{P} 3 / \mathrm{CPPM}$, Marseille, France

${ }^{h}$ Indiana University, Bloomington IN, USA

${ }^{i}$ American Astronomical Society, Washington DC, USA

${ }^{j}$ California Institute of Technology, Pasedena CA, USA

${ }^{k}$ Space Telescope Science Institute, Baltimore MD, USA

${ }^{l}$ Case Western Reserve University, Cleveland OH, USA

${ }^{m}$ CNRS/INSU/LAM, Marseille, France

${ }^{n}$ Cambridge University, Cambridge, UK

${ }^{o}$ Goddard Space Flight Center, Greenbelt MD, USA

${ }^{p} \mathrm{CNRS} / \mathrm{IN} 2 \mathrm{P} 3 / \mathrm{IPNL}$, Lyon, France

\begin{abstract}
The Supernova / Acceleration Probe (SNAP) is a proposed space-borne observatory that will survey the sky with a widefield optical/near-infrared (NIR) imager. The images produced by SNAP will have an unprecedented combination of depth, solid-angle, angular resolution, and temporal sampling. For 16 months each, two 7.5 square-degree fields will be observed every four days to a magnitude depth of $A B=27.7$ in each of the SNAP filters, spanning 3500-17000 Co-adding images over all epochs will give $A B=30.3$ per filter. In addition, a 300 square-degree field will be surveyed to $A B=28$ per filter, with no repeated temporal sampling. Although the survey strategy is tailored for supernova and weak gravitational lensing observations, the resulting data will support a broad range of auxiliary science programs.
\end{abstract}

Keywords: Astronomical imaging, wide-field surveys

Send correspondence to A. Kim: E-mail: agkim@lbl.gov 


\section{INTRODUCTION}

The unexpected discovery that the Universe's expansion is accelerating, as measured by supernova experiments ${ }^{1,2}$ and independently confirmed by cosmic-microwave-background experiments, ${ }^{3,4}$ implies that some heretofore unknown form of energy is driving the Universe's dynamics. The existence of this so-called "dark energy" lies beyond the current framework of elementary particles and thus has profound implications for fundamental physics. The challenge now is to measure the physical properties of this dark energy; the most well-developed approach is with a next generation highredshift supernova search and discovery experiment.

The Supernova / Acceleration Probe (SNAP) is proposed in this spirit. As a dedicated space-borne observatory, SNAP will provide supernova data, in the form of light curves and spectra, of unprecedented quality. The photometric instrumentation suite is tailored specifically for the needs of the supernova program; a wide-field imager in the optical and NIR provides a high discovery rate of $z<1.7$ Type Ia supernovae (SNe Ia) and allows for multiplexed followup observations, $\sim 50$ supernovae within a single exposure. The supernova fields will be revisited every four days for 16 months, providing light curves for at least several months in the rest frame of each supernova. The optical light of the supernova will observed using filter set spanning $3500-17000 \AA$ in the observer frame.

Also part of the SNAP primary mission is a weak gravitational lensing survey. Lensing provides an independent and complementary measurement of the cosmological parameters through the mapping of galaxy shape distortions induced by mass inhomogeneities in the Universe. The strengths that make SNAP excellent for supernova observations apply to lensing as well; a wide-field imager in space with stable and narrow point-spread-functions can provide large survey areas, accurate shape measurements, and high galaxy angular-surface densities. The SNAP supernova fields will serve as a deep lensing field while a second larger-solid-angle field specifically tailored for lensing will be observed to a shallower depth.

In this paper, we quantify the expected depth, solid-angle, and time resolution of the SNAP SNe and weak lensing surveys. In $\S 2$ we describe the SNAP mission: the important properties of the telescope and camera as relevant to imaging and the observing cadence and exposure times of the primary SNAP science missions. The depths of the surveys naturally produced by these programs are given in $§ 3$. A sampling of possible auxiliary science that can be done with these data is given in $\S 4$ and a brief summary is given in $\S 5$.

\section{DESCRIPTION OF MISSION}

In this section, we describe the SNAP telescope and its instrumentation suite as relevant to its imaging capabilities. The observing program for the primary supernova and lensing missions are also detailed. It is based on these properties that we calculate the depth of the resulting surveys. The numbers provided here and throughout this paper are SNAP specifications that are subject to change as we work on the conceptual design.

\subsection{Telescope and Instrumentation}

The important properties of the SNAP telescope ${ }^{5}$ are given in Table 1. SNAP has a 2-m primary aperture and $16 \%$ obscuration from the full baffling. The spot sizes from ray tracing of the telescope optics are less than 0.05" RMS over the focal plane. The optical telescope assembly is composed of four silver-coated reflectors each with $98 \%$ throughput.

Table 1. Parameters of the SNAP telescope that are relevant to determining imaging capabilities.

\begin{tabular}{|c|c|c|c|c|c|c|}
\hline Telescope & $\begin{array}{c}\text { Primary } \\
\text { Aperture }(\mathrm{m})\end{array}$ & $\begin{array}{c}\text { Secondary } \\
\text { Aperture }(\mathrm{m})\end{array}$ & $\begin{array}{c}\text { Primary } \\
\text { Obscuration }\end{array}$ & $\begin{array}{c}\text { Spot Size } \\
(\operatorname{arcsec})\end{array}$ & $\begin{array}{c}\text { Throughput } \\
(@ 1 \mu \mathrm{m})\end{array}$ & $\begin{array}{c}\text { Jitter } \\
(\operatorname{arcsec})\end{array}$ \\
\hline SNAP & 2.0 & 0.4 & 0.16 & 0.05 & 0.92 & 0.02 \\
\hline
\end{tabular}

The SNAP camera ${ }^{6}$ has $f / \#=10.83$ and throughput of $70 \%$. The camera tiles 0.7 square degrees, split in area between LBNL CCD's ${ }^{7}$ and $1.7 \mu$ m cutoff HgCdTe devices. ${ }^{8}$ The detector properties are given in Table 2 . The detector pixel sizes give undersampled images; spatial resolution will be recovered by taking several dithered images for each pointing. ${ }^{9}$ When imaging, the detector noise is sub-dominant to the zodiacal background and thus has a negligible effect on the error budget. 
Table 2. Parameters of the SNAP detectors that are relevant to determining imaging capabilities.

\begin{tabular}{|c|c|c|c|c|c|}
\hline Detector & $\begin{array}{c}\text { Pitch } \\
(\mu \mathrm{m})\end{array}$ & $\begin{array}{c}\text { Read Noise } \\
\left(\mathrm{e}^{-} / \text {pixel }\right)\end{array}$ & $\begin{array}{c}\text { Dark Current } \\
\left(\mathrm{e}^{-} / \text {sec/pixel }\right)\end{array}$ & $\begin{array}{c}\text { Diffusion } \\
(\mu \mathrm{m})\end{array}$ & Peak QE \\
\hline LBNL CCD $^{7}$ & 10.5 & 4 & 0.002 & 4 & 0.92 \\
\hline HgCdTe $^{8}$ & 18 & 5 & 0.02 & 5 & 0.6 \\
\hline
\end{tabular}

The zodiacal background will be the dominant source of background light given SNAP's orbit and shielding of Earthshine. The relatively faint wavelength-dependent background toward the ecliptic poles is shown in Figure $1 .{ }^{10}$ The cosmic-ray flux of $2 \times 10^{-4} / \mathrm{sec} / \mathrm{pix}$ will make multiple measures necessary to avoid significant contamination of the images, as will be discussed in $\S 3$.

The PSF is diffraction dominated but we include effects such as CCD diffusion, telescope jitter, and the telescope spot blur. At bluer wavelengths, the contribution of these secondary blurs do have an important effect on the PSF. For our photometric measurements, it is the PSF size that determines the noise contribution of sky background.

The SNAP filter set, their shapes, number, and distribution in wavelength space, is currently under review. The set that we consider in this note consists of nine Johnson B filters logarithmically distributed in wavelength with effective wavelengths at $4400 \times 1.15^{n} \AA$ for $n \in\{0,1, \ldots, 8\}$. We have a fixed filter design; the six optical filters are uniformly distributed over the CCD's while the three NIR filters occupy equal areas of the HgCdTe devices. An individual optical filter tile subtends $2.9^{\prime} \times 2.9^{\prime}$ and an individual NIR-filter tile subtends $5.8^{\prime} \times 5.8^{\prime}$. Summing over the tiles, each optical filter covers 0.056 square degrees over 24 squares while each NIR filter covers 0.112 square degrees over 12 squares to constitute the entire 0.7 square degree field.

The detectors are arrayed in an annulus; the SNAP three-mirror-anastigmatic design has a flat pickoff mirror near the Cassegrain focus that totally vignettes the central region of the field. The $90^{\circ}$ rotational symmetry of the filter layout allows a fixed side of SNAP to always face the sun while maintaining a consistent footprint of the fields over an entire year. Figure 2 shows the layout of the imager in the focal plane, and the relative sizes and positions of the filters.

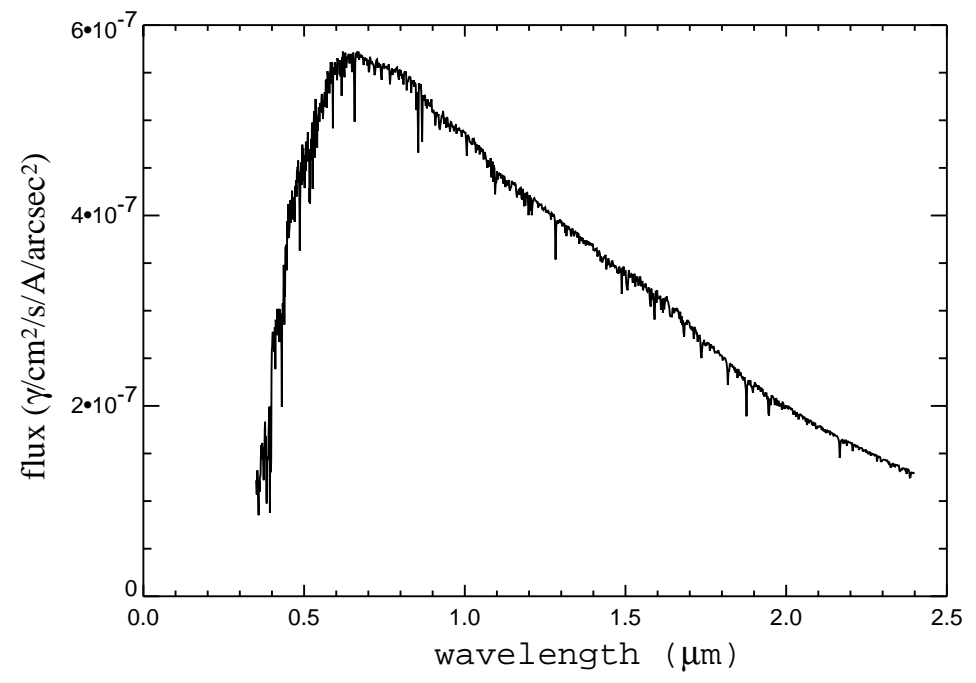

Figure 1. The zodiacal background will be the dominant source of background light for SNAP. Shown is the zodiacal photon flux toward the north ecliptic pole, the planned location for one of the SNAP supernova surveys. 


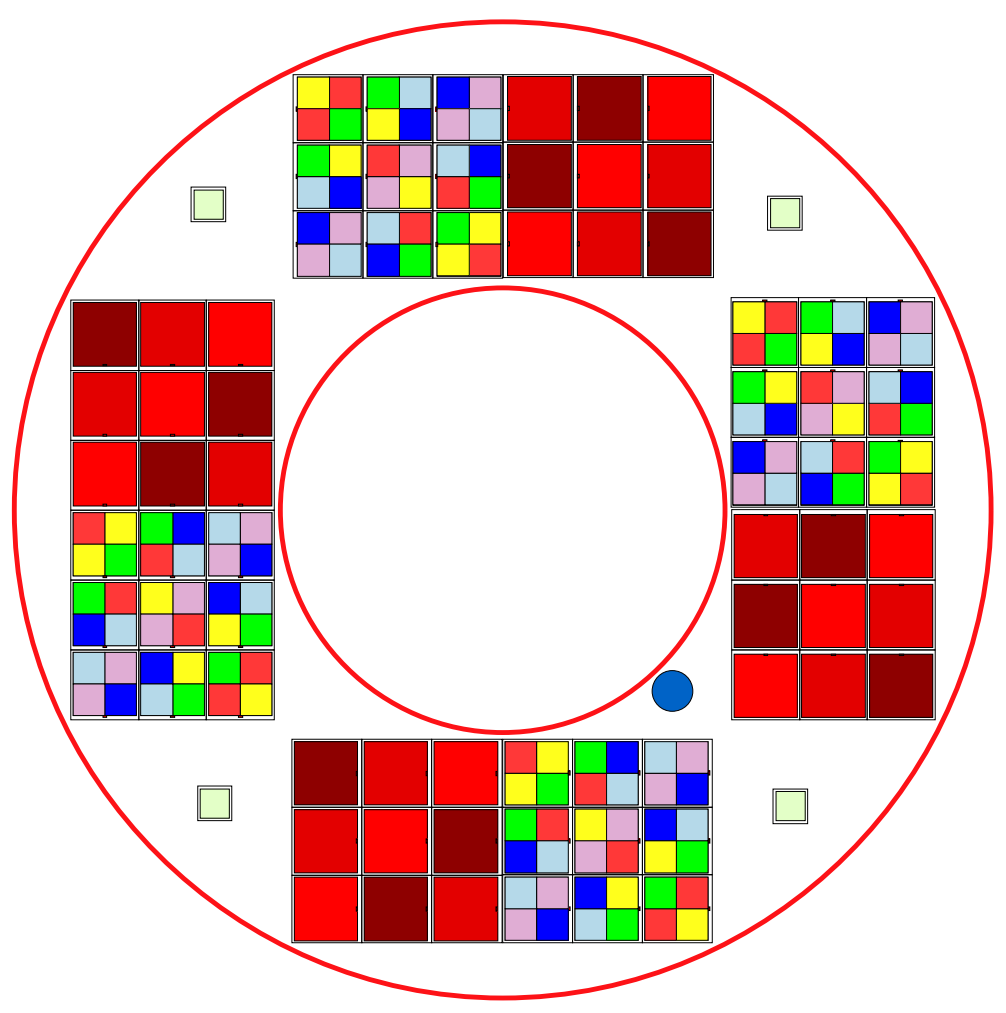

Figure 2. The layout for the SNAP imager. Detectors tile 0.7 square degrees of the focal plane. In the fixed filter scheme, six optical filters are mated to CCD's and three NIR filters are mated to HgCdTe detectors. An individual optical-filter tile subtends $2.9^{\prime} \times 2.9^{\prime}$ and an individual NIR-filter tile subtends $5.8^{\prime} \times 5.8^{\prime}$. The four star guiders are shown as isolated squares located between the science detectors. The circle at the inner part of the annulus is the light-access port for the spectrograph.

\subsection{Observing Strategy}

The two primary SNAP science programs have individually-designed observing schedules which are described here and summarized in Table 3.

\subsubsection{Supernova Program}

The supernova survey is comprised of two halves, both in time and in position. The initial survey will cover 7.5 square degrees over sixteen months pointing toward a field near the north ecliptic pole. After an intervening weak-lensing survey, a similar field toward the south ecliptic pole will be observed.

Observations in multiple bands allow SN-frame $B$ and $V$ coverage for SNe from $0<z<1.7$. As shown in Figure 2, the filters are arrayed in a checker-board pattern. In the absence of a filter wheel, multi-band exposures of a point of sky will be achieved by shift-and-stare observations where the pointings are shifted by the 2.9' width of the optical filters. Each pointing will consist of four 300-second exposures; the multiple exposures are for cosmic-ray rejection and dithering of our undersampled pixels. Within a scan, over one hundred pointings are required to cover the 7.5 square degrees in all filters to the desired depth. The wider NIR filters will experience twice the exposure time of the optical filters. A scan of the north (south) field will be repeated every four days for 16 months for a total 120 scans.

Forty percent of the SNAP mission will be spent doing targeted spectroscopy of supernovae. Imaging occurs simultaneously during these spectroscopic observations. The resulting images will cover random positions and orientations within the SNAP field and can be used to increase the depth of the survey. However, these extra images are not considered in the calculations given in this paper. 


\subsubsection{Weak Lensing Program}

The weak lensing program calls for a five-month wide-field survey to obtain as much solid-angle as possible within the constraints of telemetry. Repeat observations are unnecessary. Multi-filter data, particularly in the NIR, is desired for accurate photometric-redshift determination. The specific geometry of this field is yet to be determined. The important observing parameters of this and the supernova programs are summarized in Table 3.

Table 3. The SNAP Surveys.

\begin{tabular}{|c|c|c|c|c|}
\hline Program & $\begin{array}{c}\text { Solid Angle } \\
\text { per filter (sq. deg.) }\end{array}$ & $\begin{array}{c}\text { Exposure } \\
\text { per scan (s) }\end{array}$ & $\begin{array}{c}\text { Cadence } \\
\text { (days) }\end{array}$ & Visits \\
\hline Supernova & $7.5 \times 2$ & $\begin{array}{c}\text { Optical: } 4 \times 300 \\
\text { NIR: } 8 \times 300\end{array}$ & 4 & 120 \\
\hline Lensing & 300 & $\begin{array}{c}\text { Optical: } 4 \times 500 \\
\text { NIR: } 8 \times 500\end{array}$ & $\ldots$ & 1 \\
\hline
\end{tabular}

\section{DEPTH OF OBSERVATIONS}

The telescope and camera properties of SNAP have been modeled and incorporated into an advanced exposure-time calculator (ETC). ${ }^{9}$ Besides having all the bells and whistles of a standard ETC, our ETC includes unique handling of the pixel response function, undersampling, dithering, and probabilistic cosmic-ray rejection. As mentioned in $\S 2$, SNAP will rely on dithering to recover spatial resolution from its undersampled pixels.

The high cosmic-ray flux produces a non-trivial reduction of effective exposure times; pixels from a single exposure that are contaminated by a cosmic ray are assumed to be recognized through median filtering and dropped in the dithered reconstruction. Short individual exposure times limit the contamination: 300 second exposures give a $68 \%$ probability that there will be no cosmic-ray contamination at a given position on any of the four dithers that make up a pointing.

The magnitude depths for individual scans and co-added images of the SNAP supernova fields are calculated for each filter. The limiting magnitude for any given point is probabilistic, due to the random occurrence of cosmic rays. Table 4 shows the 50th-percentile limiting $\mathrm{AB}$ magnitude for a $S / N=5$ point source for each filter in the surveys.

Table 4. The SNAP 50th-percentile AB magnitude survey depth for a point source $S / N=5$. Random cosmic-ray hits make the $S / N$ for a given position probabilistic. The choice of filter set is currently subject to optimization studies; the filters and depths presented here are meant to be illustrative.

\begin{tabular}{|c|c|c|c|c|c|}
\hline Filter & $\lambda_{\text {eff }}(\AA)$ & $\Delta \lambda(\AA)$ & \multicolumn{2}{|c|}{ SN Survey (AB mag) } & Lensing Survey \\
\cline { 4 - 5 } & & & Scan & Co-added Scans & $($ AB mag) \\
\hline 1 & 4400 & 1000 & 27.9 & 30.6 & 28.3 \\
\hline 2 & 5060 & 1150 & 27.8 & 30.5 & 28.2 \\
\hline 3 & 5819 & 1323 & 27.8 & 30.4 & 28.1 \\
\hline 4 & 6692 & 1521 & 27.7 & 30.4 & 28.1 \\
\hline 5 & 7696 & 1749 & 27.7 & 30.3 & 28.0 \\
\hline 6 & 8850 & 2011 & 27.5 & 30.2 & 27.9 \\
\hline 7 & 10178 & 2313 & 27.5 & 30.2 & 27.8 \\
\hline 8 & 11704 & 2660 & 27.4 & 30.1 & 27.8 \\
\hline 9 & 13460 & 3059 & 27.4 & 30.0 & 27.7 \\
\hline
\end{tabular}

The SNAP observing strategy provides remarkably even depth over the range of filters. For a given filter, individual scans of the supernova and lensing surveys are only $\sim 0.75$ magnitudes shallower than the Hubble Deep Fields (HDFs) while the SN fields co-added over time are $\sim 1.5$ magnitudes deeper than the HDFs. ${ }^{11}$ SNAP has the additional advantage 
of having nine filters observing to this depth, compared to the four filters of the HDFs, and 9000 times the area; when these data are combined, the limiting magnitude increases by 0.6 magnitudes.

SNAP fields will contain many faint diffuse galaxies whose detection is important for the weak-lensing survey, and for other potential science projects. The limiting magnitudes for Gaussian-aperture photometry of an exponential-disk galaxy with FWHM=0.12" are shown in Table 5.

Table 5. The SNAP AB magnitude survey depth for an exponential-disk galaxy with FWHM=0.12" with $S / N=10$. The choice of filter set is currently subject to optimization studies; the filters and depths presented here are meant to be illustrative.

\begin{tabular}{|c|c|c|c|c|c|}
\hline Filter & $\lambda_{\text {eff }}(\AA)$ & $\Delta \lambda(\AA)$ & \multicolumn{2}{|c|}{ SN Survey (AB mag) } & Lensing Survey \\
\cline { 4 - 5 } & & & Scan & Co-added Scans & (AB mag) \\
\hline 1 & 4400 & 1000 & 26.4 & 29.1 & 26.8 \\
\hline 2 & 5060 & 1150 & 26.3 & 29.0 & 26.7 \\
\hline 3 & 5819 & 1323 & 26.3 & 29.0 & 26.6 \\
\hline 4 & 6692 & 1521 & 26.2 & 28.9 & 26.6 \\
\hline 5 & 7696 & 1749 & 26.3 & 28.9 & 26.6 \\
\hline 6 & 8850 & 2011 & 26.2 & 28.8 & 26.5 \\
\hline 7 & 10178 & 2313 & 26.3 & 28.9 & 26.6 \\
\hline 8 & 11704 & 2660 & 26.2 & 28.9 & 26.6 \\
\hline 9 & 13460 & 3059 & 26.2 & 28.9 & 26.5 \\
\hline
\end{tabular}

\section{SCIENCE}

In this section we give a brief discussion of possible science that can be obtained from the SNAP surveys. This list is by no means complete in its breadth nor depth. The expected results from the primary SNAP science missions are discussed in a companion paper. ${ }^{12}$

The Sloan Digital Sky Survey(SDSS) ${ }^{13}$ and HDFs ${ }^{11,14}$ have demonstrated the vast range of science that can be obtained from wide and deep multi-band surveys. SNAP will produce surveys that dwarf the 0.0016 square degrees size of the HDFs and go even deeper with time-sampling for its supernova fields. The SNAP lensing field is about the same size as the Sloan Southern Survey and CFHT Legacy Survey fields but several magnitudes deeper. This combination of depth, temporal coverage, filter coverage over a broad wavelength range, diffraction-limited seeing, and wide field make SNAP imaging surveys uniquely powerful in the study of a wide range of objects:

- Galaxies - SNAP fields will contain $>5 \times 10^{7}$ galaxies within detection threshold. Statistical studies are possible with such a large sample, e.g. the determination of the galaxy luminosity function and color distributions as a function of redshift. The depth allows discovery of low-surface-brightness and very high-redshift galaxies. Accurate photometric redshifts and information on galaxy evolution will be available from the multi-band and in particular the NIR photometry. High-resolution images will provide a view of the internal structure of galaxies and their kinematic interactions with each other.

- Galaxy clusters - Wide-field imaging and photometric redshifts allow easy identification of galaxy clusters. The epoch of galaxy-cluster formation is tightly linked with the mass density of the Universe, $\Omega_{M}$, providing an independent cosmological measurement complementary to SNAP's primary missions.

- Quasars - The NIR photometry extends the redshift range for quasar discovery $(6.3<z<12)$ using colors and dropout surveys. Discoveries will also move much fainter into the quasar luminosity function. The highest redshift quasars can be used to map the reionization history of the Universe through the Gunn-Peterson effect. SNAP's ability to identify diffuse objects associated with quasars may present many opportunities for the study of galaxy formation. 
- Transients/Variables - The discovery and observation of SNe Ia are the primary goals of SNAP, but transient "backgrounds" are interesting in their own right: quasars, active-galactic-nuclei, gamma-ray-burst optical counterparts, supernovae of other types, variable stars, and eclipsing binaries. Of particular interest to cosmology is time-delay studies with the expected large number of strongly lensed variables. Gravitational microlensing surveys of stars and quasars to measure dark matter are also possible.

- Stars - Faint limiting magnitudes and excellent star-galaxy separation will yield faint dwarf and halo stars. Proper motion can be detected with high-resolution and a long time baseline. The geometry and substructure of the halo and disk in the direction of the SNAP fields can be mapped.

- Solar-system objects - The peculiar motion in the time-series data will facilitate the identification of local objects such as asteroids and Kuiper-belt objects.

The output from the SDSS has demonstrated how the natural byproducts of a wide-field survey can produce scientific yield well beyond the scope of its primary purpose. Individual objects found on SDSS images are routinely observed spectroscopically at the largest telescopes in the world, fulfilling the historical trend of small-aperture telescope imaging feeding targets for large-aperture telescope spectroscopy. The SNAP surveys will provide a similar opportunity in working with NGST and the next generation of ground-based wide-aperture telescopes.

\section{SUMMARY}

As we have discussed, SNAP will provide a combination of depth, solid-angle, angular resolution, and temporal sampling heretofore unachieved. The primary science missions of SNAP will provide survey fields in filters spanning 3500 $17000 \AA$.

- A 300 square-degree field to $\mathrm{AB}$ mag $\sim 28$ at $S / N=5$ in each filter.

- Two 7.5 square-degree fields observed every four days 120 times in all filters. Each observation reaches AB mag $\sim 27.7$ at $S / N=5$ in each filter.

- The co-added sum of all visits from the preceding survey: two 7.5 square-degree fields to $\mathrm{AB}$ mag $\sim 30.3$ at $S / N=5$ in each filter.

We have restricted our discussion to surveys produced by the principal science missions of SNAP* Additional imaging surveys will be produced from data taken after completion of the SNAP primary missions; a guest observing program is envisioned filling the remaining satellite lifetime to allow the full potential of SNAP to be realized.

\section{ACKNOWLEDGMENTS}

This work was supported by the Director, Office of Science, of the U.S. Department of Energy under Contract No. DEAC03-76SF00098.

\section{REFERENCES}

1. S. Perlmutter et al. ApJ 517, p. 565, 1999.

2. A. Riess et al. AJ 116, p. 1009, 1998.

3. A. Balbi et al. ApJ 545, pp. L1-L4, 2000.

4. A. Lange et al. PRD , p. 042001, 2001.

5. M. Lampton et al., "SNAP Telescope," in Survey and Other Telescope Technologies and Discoveries, Proceedings of SPIE 4849, 2002.

${ }^{*}$ The numbers presented here describe the SNAP reference mission. They are subject to change as trade studies are performed to optimize the design of the experiment. The reader is advised to exercise caution in extrapolating these results to other possible survey missions; the SNAP primary-mission fields have continuous visibility, allow for stable satellite orientation, ensure shielding of background light, and point to regions of relatively low zodiacal background. 
6. M. Lampton et al., "SNAP Focal Plane," in Survey and Other Telescope Technologies and Discoveries, Proceedings of SPIE 4854, 2002.

7. D. Groom et al. NIM A442, p. 216, 2000.

8. G. Tarlé et al., "The SNAP Near Infrared Detectors," in Survey and Other Telescope Technologies and Discoveries, Proceedings of SPIE 4850, 2002.

9. G. Bernstein PASP 114, pp. 98-111, 2002.

10. G. Aldering, "SNAP Sky Background at the North Ecliptic Pole," LBNL report (LBNL-51157), 2002.

11. R. E. Williams et al. AJ 112, p. 1335, 1996.

12. G. Aldering et al., "Overview of the SuperNova / Acceleration Probe," in Survey and Other Telescope Technologies and Discoveries, Proceedings of SPIE 4835, 2002.

13. D. G. York et al. AJ 120, pp. 1579-1587, 2000.

14. R. E. Williams et al. AJ 120, pp. 2735-2746, 2000. 